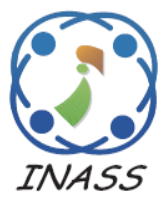

\title{
A Novel FOC Vector Control Structure Using RBF Tuning PI and SM for SPIM Drives
}

\author{
Ngoc Thuy Pham ${ }^{1 *}$ \\ Thuan Duc Le ${ }^{1}$ \\ ${ }^{I}$ Department of Electrical Engineering Technology, Industrial University of Ho Chi Minh City, Vietnam \\ * Corresponding author's Email: ngocpham1020@gmail.com
}

\begin{abstract}
This paper presents a new structure combining (RBF PI) RBF neural network tuning PI and (SM) Sliding Mode nonlinear control for the FOC vector control of the (SPIM) six phase induction motor drives. In this new scheme, SM control is proposed for inner current loop to control better current of SPIM. The improved adaptive PI controller is proposed for outer speed loop, in this proposal, the mathematical model of SPIM has been identified via the RBF neural networks, and then the PI parameters can be optimized automatically to accommodate the characteristic variation of the process. The performance of the proposed speed control scheme is validated by Matlab-Simulink software. The obtained simulation results have confirmed that the torque and rotor flux ripples are significantly minimized for entire speed range. The proposed RBFNN based on PI_SM controller give accurate speed control, fast dynamics response, the performance, stability and robustness of SPIM drive system have been improved significantly.
\end{abstract}

Keywords: RBF neural network tuning PID control, SM control, Six phase induction motor drives, FOC vector control.

\section{Introduction}

In recent decades, the multiphase motor drives are widely used in many applications due to their inherent features such as higher torque density, greater efficiency, reduced torque pulsations, fault tolerance, and reduction in the required rating per inverter leg [1]. Especially, these drives are often considered in some applications such as locomotive traction, electrical ship propulsion, in high power applications such as automotive, aerospace, military and nuclear [2]. With its reliable working characteristics and high failure tolerance nowadays, these motors are even considered in the small power applications requiring high reliability and fault tolerance, where are expected that the loss of one or more phases the machine still can provide a significant electromagnetic torque to continue operating the system. Among the many types of multiphase motors, SPIM is one of the most widely used multiphase motors.
In traditional FOC vector control of SPIM drives, the PID controller is widely applied due to its relative simple implementation and effectiveness. However, with the $\mathrm{Ki}, \mathrm{Kp}, \mathrm{Kd}$ fixed gains, the PID controller does not satisfy the requirements of the high performance SPIM drives. Therefore, recently the development of the modern vector control strategies based on the new controller has received great attention. Like the feedback linearizing technique in $[3,4]$, the technique of feedback linearizing based on the differential geometry allows by a diffeomorphic transformation and a state feedback. The feedback linearization approach to design a controller to achieve input-output decoupling, high dynamic performance, and high power efficiency. It cancels the nonlinear terms in the machine model. However, the parametric deviation will significantly affect the dynamic performance and the stability for practical implementation. By contrast, the passivity based control [5] doesn't cancel all the nonlinearity but ensure system stability, by adding a damping term to 
the total energy of the system. It is characterized by its robustness for the parameter uncertainties, but its experimental implementation is still difficult. Backstepping controlled approach $[6,7]$ is based on the Lyapunov stability theories. This approach offers great flexibility in the synthesis of the regulator and it also has the flexibility to avoid cancelations of useful nonlinearities and pursues the objectives of stabilization and tracking. However, the information of detailed and accurate system dynamics was required in the traditional BS scheme, which is difficult to obtain in practical application. In another approach, the fuzzy logic controller is used to deal with the unknown nonlinear functions and uncertain parameters. This is because FL controller does not require a mathematical model and it is capable of handling linear and non-linear systems and generates human logic linguistic rules [7, 8]. However, the performance of FL controller depends on its input and output membership functions. Neural networks controller also (NN) has been successfully used for the control of dynamic system and identification $[9,10]$. It is easy to design nonlinear controllers with the ability NN modeling of nonlinear dynamical systems. The high computation rate, learning, and adaption capability of $\mathrm{NN}$ makes them ideal for adaptive control systems. However, using $\mathrm{NN}$ to enhance the performance of the controller will require the computational burden compare to the conventional cotrollers. Finally, one of the most common nonlinear control methods today is the sliding mode control. This is characterized by simplicity of design and attractive robustness properties. Its major drawback is the chattering phenomenon [11, 12]. From the above analysis, we see that these nonlinear control techniques are usually quite complex, demanding high computational effort, and requiring a precise mathematical model. They were difficult to obtain satisfactory control performance when using independently, especially in the cases applied to control the nonline systems. Therefore, recently the combination of the different control techniques to both simple and enhance the performance of SPIM drives is a hot topic in the motor control field. Like in [15], the fuzzy sliding mode controller has proposed reduce the phenomena chattering. However, the overshoot of the torque, stator current and rotor flux have increased highly in transient modes reported.

In this paper, the author proposes a new combined control structure: The RBF NN based on PI and SM controller. As we known, the design of FOC vector control system for high-performance SPIM drives has complied with the basic design principle of FOC consisting of two loops, First, requiring with the inner current loop are fast response and highly robust and stable. Second, requiring with the outer speed and rotor flux loop are slower response. The fast response and stable internal current loop are critical to ensure the quality of control for a high performance SPIM drive. To meet these control criteria, internal current controller is proposed using sliding mode control. The success of this SM type of control for electric drives are mainly due to its disturbance rejection, strong robustness, fast response and simple implementation, as shown by large number of papers on AC drives, that use the standard approach of SM control [11, 12]. In this paper, sliding mode controller is proposed for the inner current closed loop control to can effectively compensate for load disturbance in the system so the proposed method is more robust, stability and faster dynamics response. In contrast, unlike the current controllers, the speed response depends on the inertia of the machine. Therefore, if a fast acting controller is used into the outer speed and rotor flux of FOC vector control of SPIM drives, it can always provide isq reference current component in excess the allowed limit and then the controllers will default work at the critical value. In other words, the output of the speed and rotor loop always work on saturated modes at the limit values, the working time in these modes depend on the sudden change in reference speed and the time constant due to inertia mechanics of the machine. These make the system lost control whenever the controller reaches saturation. To overcome this, the slow-acting PI controller is proposed instead of the inappropriate fast-acting controller, this option is quite good from a mechanical mechanic point of view. As you know, in the industrial control field, the PID controller has been the most commonly used controller. Its advantages are simple, stable, robust and easy for implementation [15]. The advanced nonlinear control methods are quite complicated and difficult to implement on the real industries. However, the conventional Proportional Integral-Derivative (PID) control scheme with fixed parameters has been difficult to obtain satisfactory control performance. To address the above mentioned problem, one possible way is to adjust the conventional PID controller structure, and make the parameters of the PID controller to be automatic tuning. The Radial Basis Function (RBF) neural network, which is able to approach a nonlinear function arbitrarily [16], can be used to identify online the mathematical model of SPIM with high accuracy, and then the PID parameters can be optimized automatically base on 
RBF to diverse the characteristic variation of the operating process.

This novel RBF NN based on PI_SM nonlinear combination structure ensure stability, robustness and accurate speed control of SPIM drives. The effectiveness of this proposed control structure is verified by simulation using MATLAB/ Simulink. The paper is organized into five sections, in section 2 , the basic theory of the model of the SPIM and the SPIM drive are presented. Section 3 introduces the proposed RBF NN tuning PI _SM controller. Simulation and discuss are presented in section 4. Finally, the concluding is provided in section 5.

\section{Model of SPIM drives}

The system includes the six phase induction motor fed by a six-phase Voltage Source Inverter (SPVSI) and a DC link. A diagram of the SPIMD is illustrated as in Fig. 1. In this part, the Vector Space Decomposition (VSD) technique also has applied as in [15], the original six-dimensional space of the machine is transformed into three two-dimensional orthogonal subspaces in the stationary reference frame (D-Q), (x - y) and (zl -z2). This transformation is obtained by means of $6 \times 6$ transformation matrix [2]:

$$
T_{6}=\frac{1}{3}\left[\begin{array}{cccccc}
1 & \frac{\sqrt{3}}{2} & -\frac{1}{2} & -\frac{\sqrt{3}}{2} & -\frac{1}{2} & 0 \\
0 & \frac{1}{2} & \frac{\sqrt{3}}{2} & \frac{1}{2} & -\frac{\sqrt{3}}{2} & -1 \\
1 & -\frac{\sqrt{3}}{2} & -\frac{1}{2} & \frac{\sqrt{3}}{2} & -\frac{1}{2} & 0 \\
0 & \frac{1}{2} & -\frac{\sqrt{3}}{2} & \frac{1}{2} & \frac{\sqrt{3}}{2} & -1 \\
1 & 0 & 1 & 0 & 1 & 0 \\
0 & 1 & 0 & 1 & 0 & 1
\end{array}\right]
$$

To build SPIM model, some basic assumptions should be made. First, the windings are seen as to be sinusoidal distribution, the mutual leakage inductances, the magnetic saturation, and the core losses are neglected. The math equations of SPIM be written in the stationary reference frame as

$$
\begin{aligned}
{\left[V_{s}\right] } & =\left[R_{s}\right]\left[I_{s}\right]+P\left(\left[L_{s}\right]\left[I_{s}\right]+\left[L_{m}\right]\left[I_{r}\right]\right) \\
0 & =\left[R_{r}\right]\left[I_{r}\right]+P\left(\left[L_{r}\right]\left[I_{r}\right]+\left[L_{m}\right]\left[I_{s}\right]\right)
\end{aligned}
$$

where: $[\mathrm{V}],[\mathrm{I}],[\mathrm{R}],[\mathrm{L}]$ and $[\mathrm{Lm}]$ are voltage, current, resistant, self and mutual inductance vectors, respectively. $\mathrm{P}$ is differential operator. Subscript $\mathrm{r}$

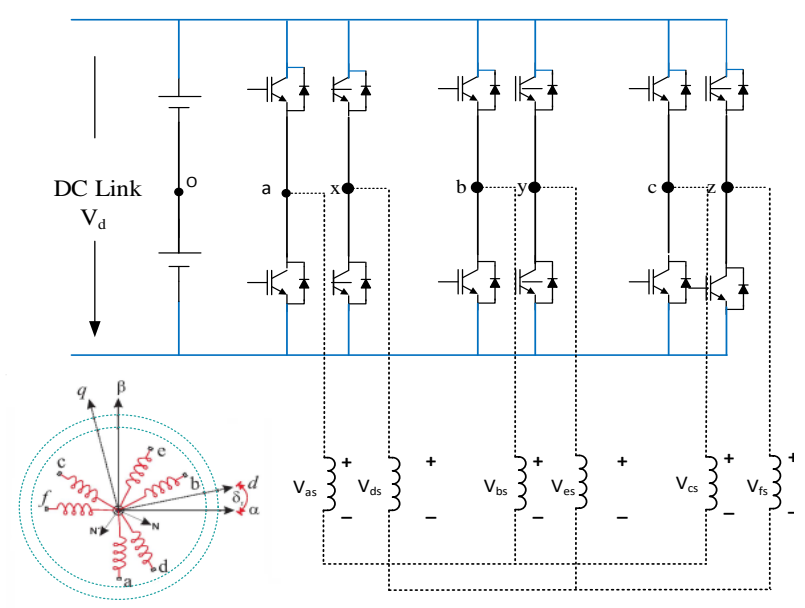

Figure. 1 A SPIM drive general diagram

and $\mathrm{s}$ related to the rotor and stator resistance respectively. Since the rotor is squirrel cage, $[\mathrm{Vr}]$ is equal to zero. The electromechanical energy conversion only takes place in the DQ subsystem. The torque equation can be written as follows:

$$
T_{e}=3 n_{p}\left(\psi_{r Q} i_{r D}-\psi_{r D} i_{r Q}\right)
$$

where: respectively, Te, np, $\Psi r D, \Psi r Q$, irD, irQ are the electromagnetic torque that generated by the motor, number of pairs of poles, the rotor flux, rotor current, respectively.

As you knew, (x - y) and (zl -z2) subspace produced losses, the electromechanical conversion just takes place in the D-Q subspace [2]. Therefore, the control is based on determining the applied voltage in the DQ reference coordinates. Then the SPIM control technique is similar to the classical three phase induction motor. The control for the motor in the stationary reference coordinates is difficult, even for a three phase IM, so the transformation of SPIM model in a $\mathrm{dq}$ rotating reference coordinates to obtain currents with dc components is necessary, a transformation matrix must be used to represent the stationary reference coordinates (DQ) in the dynamic (dq) rotating reference coordinates. This matrix is given:

$$
T_{d q}=\left[\begin{array}{cc}
\cos \left(\delta_{r}\right) & -\sin \left(\delta_{r}\right) \\
\sin \left(\delta_{r}\right) & \cos \left(\delta_{r}\right)
\end{array}\right]
$$

where $\delta \mathrm{r}$ is the rotor angular position referred to the stator as shown in Fig. 1.

FOC is one of the most common control methods, Unlike the scalar control, FOC can improve the static and dynamic behavior of SPIM. FOC control can control torque and magnetic flux 
separately as the control way to DC motor. In that, the electromagnetic torque will be controlled by the isq stator current component, the rotor flux will be controlled by the isd stator current component. We have: $\psi_{\mathrm{rq}}=0, \psi_{\mathrm{rd}}=\psi_{\mathrm{rd}}$. Using Eqs. (1) and (4), the new dynamics model of motor is described by the space vector differential equations:

$$
\left\{\begin{array}{l}
\frac{d \omega}{d t}=\frac{3}{2} n_{p} \frac{\delta \sigma L_{s}}{J}\left(\psi_{r d} i_{s Q}\right)-\frac{T_{L}}{J}-B \omega \\
\frac{d \psi_{r d}}{d t}=\frac{L_{m}}{\tau_{r}} i_{s D}-\frac{1}{\tau_{r}} \psi_{r d} \\
L_{s} \frac{d i_{s D}}{d t}=-a i_{s D}+L_{s} \omega_{s} i_{s Q}+b R_{r} \psi_{r d}+c u_{s D} \\
L_{s} \frac{d i_{s Q}}{d t}=-a i_{s Q}+L_{s} \omega_{s} i_{s D}+b_{r} \omega_{r} \psi_{r d}+c u_{s Q}
\end{array}\right.
$$

where

$$
\sigma=1-\frac{L_{m}^{2}}{L_{s} L_{r}} ; \delta=\frac{L_{m}}{\sigma L_{s} L_{r}} ; a=\frac{L_{m}^{2} R_{r}+L_{r}^{2} R_{s}}{\sigma L_{r}^{2}} ; c=\frac{1}{\sigma} ; b=\frac{L_{m}^{2} R_{r}}{\sigma L_{r}^{2}} ; \tau_{r}=\frac{L_{r}}{R_{r}}
$$

$u_{s d}, u_{s q} ; i_{s d}, i_{s q}$ : The components of stator voltage and stator current, respectively; $\psi_{r d}, \psi_{r q}$ : Rotor flux components; $T_{e}, T_{L}$ : Electromagnetic and load torque; $d-q ; D-Q$ : Synchronous and stationary axis reference frame quantities, respectively; $\omega, \omega_{\mathrm{sl}}, \omega_{\mathrm{s}}$ : Rotor and slip angular and synchronous angular velocity, respectively; $L_{s}, L_{r}$ : Stator and rotor inductances; $L_{m}$ : Mutual inductance ; $R_{s}, R_{r}::$ Stator and rotor resistances; $J$ : the inertia of motor and load; $\sigma$ : Total linkage coefficient; $n_{p}:$ Number of pole pairs; B: Friction coefficient; $\tau_{r}$ : Rotor and stator time constant

The electromagnetic torque and the sliding frequency are expressed as follows:

$$
\begin{aligned}
& T_{e}=\frac{3}{2} n_{p} \frac{L_{m}}{L_{r}} \psi_{r d} i_{s q} ; \\
& \omega_{s l}=\frac{L_{m}}{\tau_{r} \psi_{r q}} i_{s q}
\end{aligned}
$$

\section{The proposed RBF NN based on PI _SM cotroller for vector control of SPIM drives}

\subsection{The proposed RBF NN based on PI controller for outer speed control}

\subsubsection{RBF neural network}

RBF neural network is a three-layer feedforward neural network (The RBF neural networks configuration for the speed controller is shown in Fig. 2.) with single hidden layer. Although the hidden layer to output layer is linear. However, the mapping from input to output is nonlinear. RBF network has been validated the ability of approximating any continuous function with any arbitrary accuracy. For this NN, the problem of local minimum has been eliminated and the learning rate also has made quickened greatly.

Suppose the input vector of the RBF neural networks is $x=\left[x_{1}, x_{2}, \ldots x_{n}\right]^{T}$; the radial vector is $h=\left[h_{1}, h_{2}, \ldots . h_{n}\right]^{T}$; where $h_{j}$ is Gaussian function with the following mathematical relation:

$$
h_{j}=\exp \left(-\frac{\left\|X-C_{j}\right\|^{2}}{2 b_{j}^{2}}\right) \quad(j=1,2, \ldots, m)
$$

where, the center vector of the network at node $\mathrm{j}$, the radial width vector and weight vector $\mathrm{W}$ of the network: $c_{j}=\left[\begin{array}{llll}c_{j 1}, & c_{j 2}, \ldots . & c_{j m},\end{array}\right]^{T}$. The radial basis width vector B and are: $b=\left[b_{1}, b_{2}, \ldots . b_{m},\right]^{T}$ where bj is the basis width parameter of node, and is greater than zero; The weight vector of the network is $w$ and $w=\left[w_{1}, w_{2}, \ldots j m,\right]^{T}$. Network output is

$$
y_{m}(k)=w_{1} h_{1}+w_{2} h_{2}+\ldots+w_{m} h_{m}
$$

Defining a performance index function is

$$
E(k)=\frac{1}{2}\left[y(k)-y_{m}(k)\right]^{2}
$$

According to the gradient descent method, the iterative algorithm of weight output, node center and radial basis width parameters can be written as:

$$
\begin{aligned}
& \Delta w_{j}(k)=-\eta\left[y(k)-y_{m}(k)\right] h_{j} \\
& \Delta b_{j}=\left[y(k)-y_{m}(k)\right] w_{j} h_{j} \frac{\left\|X-C_{j}\right\|^{2}}{b_{j}^{3}} \\
& \Delta c_{j i}(k)=\left[y_{\text {out }}(k)-y_{m}(k)\right] w_{j} \frac{x_{j}-c_{j i}}{b_{j}^{2}}
\end{aligned}
$$


We have

$$
\begin{array}{r}
w_{j}(k)=w_{j}(k-1)+\Delta w+\alpha\left[w_{j}(k-1)-w_{j}(k-2)\right] \\
b_{j}(k)=b_{j}(k-1)+\eta \Delta b_{j}+\alpha\left[b_{j}(k-1)-b_{j}(k-2)\right]
\end{array}
$$

$$
c_{j i}(k)=c_{j i}(k-1)+\eta \Delta c_{j i}+\alpha\left[c_{j i}(k-1)-c_{j i}(k-2)\right]
$$

where $\eta$ is learning rate, $\alpha$ is momentum factor The Jacobian algorithm is

$$
\frac{\partial y(k)}{\partial \Delta u(k)} \approx \frac{\partial y_{m}(k)}{\partial \Delta u(k)}=\sum_{j=1}^{m} w_{j} h_{j} \frac{c_{j i}-x_{1}}{b_{j}^{2}}
$$

\subsubsection{RBFNN based PID controller}

The incremental PI controller is adopted. The control error is:

$$
\varepsilon(k)=y^{*}(k)-y_{m}(k)=\omega_{r}^{*}(k)-\omega_{r}(k)
$$

The inputs of the controller are as follows:

$$
\begin{aligned}
& x c(1)=\varepsilon(k)-\varepsilon(k-1) \\
& x c(2)=\varepsilon(k)
\end{aligned}
$$

Then the incremental PI control algorithm is

$$
\Delta u(k)=k_{p}[x c(1)]+k_{I}[x c(2)]
$$

Neural network-tuning of indicators is as follows:

$$
E(k)=\frac{1}{2} \varepsilon^{2}(k)
$$

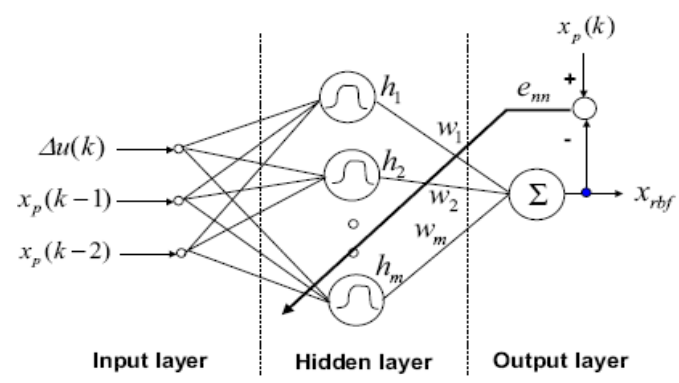

Figure. 2 The structure of the RBF NN

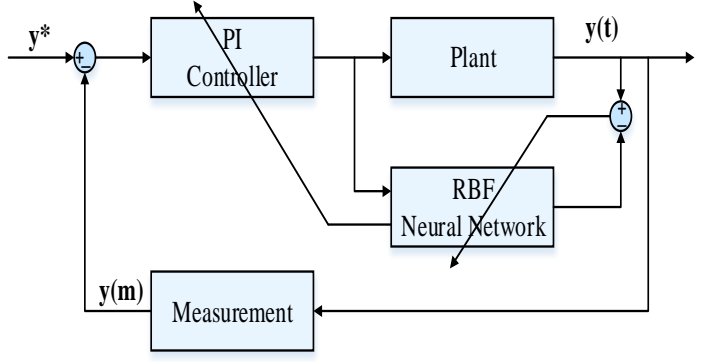

Figure. 3 The structure of the PID control system based on the RBF neural networks

The control parameters of the PI controller are adjusted based on the gradient descend method are as follows:

$$
\left\{\begin{array}{l}
\Delta k_{P}=-\eta \frac{\partial E}{\partial k_{p}}=-\eta \frac{\partial E}{\partial y} \frac{\partial y}{\partial \Delta u} \frac{\partial \Delta u}{\partial k_{p}}=\eta \varepsilon(k) \frac{\partial y}{\partial \Delta u} x c(1) \\
\Delta k_{I}=-\eta \frac{\partial E}{\partial k_{I}}=-\eta \frac{\partial E}{\partial y} \frac{\partial y}{\partial \Delta u} \frac{\partial \Delta u}{\partial k_{I}}=\eta \varepsilon(k) \frac{\partial y}{\partial \Delta u} x c(2)
\end{array}\right.
$$

where $\partial y / \partial \Delta u$ is the Jacobian information in Eq. (17), and it can be identified by the RBF neural networks. The structure of PID controller based on the RBF neural networks is shown in Fig. 3.

\subsection{SMC design for in the inner current loops}

In this paper, the inner current control objective is to make the measured stator currents $i_{s d} ; i_{s q}$ reach the desired currents value $i_{s d}^{*} ; i_{s q}^{*}$. SMC is adopted for the currents loop of SPIM drives, anch adaptation law for this SMC scheme is derived based on Lyapunov theory to ensure stability and fast error dynamics.

Defining the current tracking errors as:

$$
\varepsilon_{i_{s d}}=i_{s d}^{*}-i_{s d} ; \varepsilon_{i_{s q}}=i_{s q}^{*}-i_{s q}
$$

Consider s1 and s2 are the two sliding surfaces are defined for isd and isq, respectively. These sliding surfaces can be described as

$$
\begin{aligned}
& s_{1=} \varepsilon_{i_{s d}}+k_{1} \int_{0}^{t} \varepsilon_{i_{s d}} d t \\
& s_{2=} \varepsilon_{i q}+k_{2} \int_{0}^{t} \varepsilon_{i_{s q}} d t
\end{aligned}
$$

where $\mathrm{k} 1$ and $\mathrm{k} 2$ are the undetermined coefficient. The time derivative of Lyapunov function yields: 


$$
\begin{aligned}
& \dot{s}_{1}=\dot{\varepsilon}_{i_{s d}}+k_{1} \varepsilon_{i_{s d}} \\
& \dot{s}_{2}=\dot{\varepsilon}_{i q}+k_{2} \varepsilon_{i_{s q}}
\end{aligned}
$$

Substituting Eq. (5) to Eqs. (26)-(27) and combining to the sliding mode exponential approach law, we get the following equation:

$$
\begin{array}{r}
\dot{s}_{1}=\dot{\varepsilon}_{i_{s d}}+k_{1} \varepsilon_{i_{s d}}=i_{s d}^{*}+\frac{1}{L_{s}}\left[a i_{s d}-L_{s} \omega_{s} i_{s q}\right. \\
\left.-b R_{r} \psi_{r d}-c u_{s d}\right]+k_{1} \varepsilon_{i_{s d}}=u_{1} \\
\dot{s}_{2}=\dot{\varepsilon}_{i q}+k_{2} \varepsilon_{i_{s q}}=i_{s q}^{*}+\frac{1}{L_{s}}\left[a i_{s q}-L_{s} \omega_{s} i_{s d}-\right. \\
\left.b_{r} \omega_{r} \psi_{r d}-c u_{s D}\right]+k_{2} \varepsilon_{i_{s q}}=u_{2}
\end{array}
$$

where, $\alpha 1, \alpha 2, \beta 1, \beta 2$ are positive constants and the sign function is defined as:

$\operatorname{sign}\left(\mathrm{s}_{i}\right)=\left\{\begin{array}{lll}-1 & \text { for } & \mathrm{s}_{i}<0 \\ +1 & \text { for } & \mathrm{s}_{i}>0\end{array}\right\} ; \quad \mathrm{i}=1,2$

The SM control law can be found using Lyapunov theory and defining the Lyapunov function candidate:

$$
V=\frac{1}{2}\left(\mathrm{~s}_{1}^{2}+s_{2}^{2}\right)
$$

The time derivative of Lyapunov function can be calculated as:

$$
\dot{V}=s_{1} \dot{s_{1}}+s_{2} \dot{s_{2}}
$$

According to Lyapunov theory, if the function $V^{\prime}$ is negative definite, this will ensure that the state trajectory will be driven and attracted toward the sliding surface $s$ and once reached, it will remain sliding on it until the origin is reached asymptotically. Combining Eqs. (26) - (30), and the condition to satisfy the stability of the inner loops, the reference voltage of the stator $d$ and the $q$ axis are chosen as follows:

$$
\begin{aligned}
& u_{s d}^{*}=\frac{L_{s}}{c}\left(\alpha_{1} \operatorname{sign}\left(s_{1}\right)+\beta_{1} s_{1}+i_{s d}^{*}+\frac{1}{L_{s}}\left(a i_{s d}-L_{s} \omega_{s} i_{s q}-b R_{r} \psi_{r d}\right)+k_{1} \varepsilon_{i_{s d}}\right) \\
& u_{s q}^{*}=\frac{L_{s}}{c}\left(\alpha_{2} \operatorname{sign}\left(s_{2}\right)+\beta_{2} s_{2}+i_{s q}^{*}+\frac{1}{L_{s}}\left(a i_{s q}+L_{s} \omega_{s} i_{s d}+b_{r} \omega_{r} \psi_{r q}\right)+k_{2} \varepsilon_{i_{s q}}\right)
\end{aligned}
$$

This ensure that:

$\dot{V}=-\alpha_{1} \operatorname{sign}\left(s_{1}\right) s_{1}-\beta_{1} s_{1}^{2}-\alpha_{2} \operatorname{sign}\left(s_{2}\right) s_{2}-\beta_{2} s_{2}^{2}<0$

\section{Simulink and discussion}

The IFOC vector control of SPIM drives system is simulated by using MATLAB software. The block diagram of system is shown in Fig. 3. The proposed RBF based on PI_SM controller is compared with the classical control using a PI controller and with the other latest methods in [4, 6-9, 14] to confirm the effectiveness of the proposed algorithm. The analysis results also show the characteristic robustness of the RBF based on PI_SM control to disturbances of the load, the speed variations.

SPIM parameters: $220 \mathrm{~V}, 50 \mathrm{~Hz}, 4$ pole, 1450 rpm. $\mathrm{Rs}=10.1 \Omega, \mathrm{Rr}=9.8546 \Omega, \mathrm{Ls}=0.833457 \mathrm{H}$, $\mathrm{Lr}=0.830811 \mathrm{H}, \mathrm{m}=0.783106 \mathrm{H}, \mathrm{Ji}=0.0088$ kg.m2. Rs is nominal value of stator resistance.

\subsection{Dynamic performance of the proposal controller on speed reversal}

This test is carried up to confirm the dynamic performance of the proposed algorithm. In this test, the proposed RBF based on PI_SM controller is compared to the classical controller using a classical PI controller and supervisory type-2 fuzzy controller proposed in [9, Figs. 10-13]. The obtained speed, torque, stator current and rotor flux responses are shown in Fig. 4. The speed reference in this case is set up speed reversal from $104.713 \mathrm{rad} / \mathrm{s}$ to 104.713 (equivalent $1000 \mathrm{rpm}$ to $-1000 \mathrm{rpm}$ ) at rated load for PI and RBF based on PI _ SM controllers, respectively. Initially, SPIM is accelerated in both the cases, it is easy to see that RBF based on PI _ SM controller can provide stability and fast dynamic responses, speed transient time from zero to $1000 \mathrm{rpm}$ at in the case of PI controller is $0.155 \mathrm{~s}$ and $0.098 \mathrm{~s}$ in the case of RBF based on PI _ SM controller as shown in Fig. 5, respectively. When motor runs at steady state at $1000 \mathrm{rpm}$, a step speed command of $-1000 \mathrm{rpm}$ is applied at $\mathrm{t}=1 \mathrm{~s}$. As soon as $-1000 \mathrm{rpm}$ is applied, negative torque is developed on the shaft of the rotor, consequently motor starts decelerating achieving zero speed and then starts accelerating in reverse direction and finally get in settled down at -1000 rpm. Total time taken speed reversal of the SPIM drive using PI controller is $0.13 \mathrm{~s}$, and $0.08 \mathrm{~s}$ using RBF based on PI _ SM controller, respectively. Comparison the response time of load torque dynamics of RBF based on PI _ SM and PI 


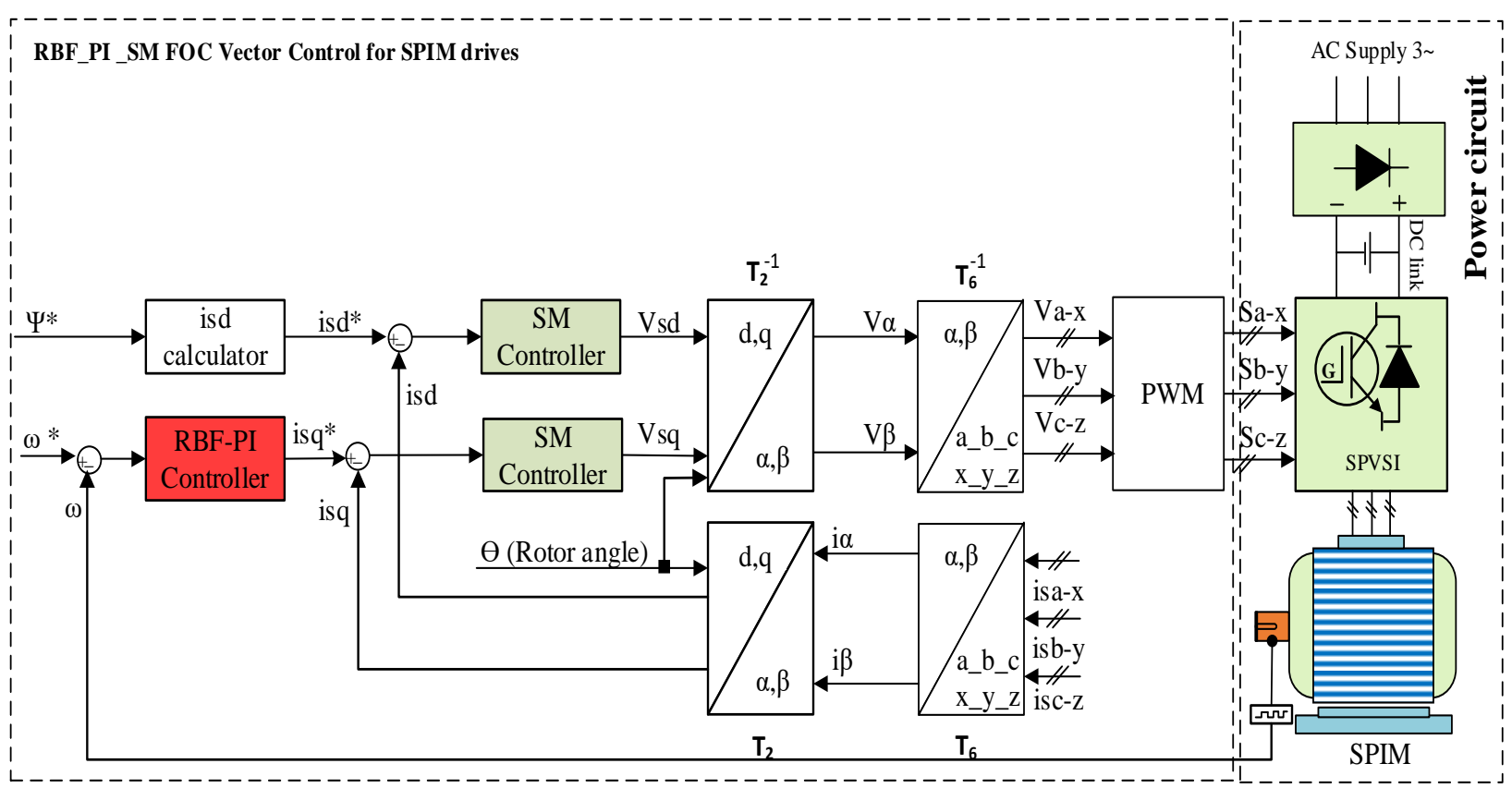

Figure. 4 Vector control of SPIM drive using RBF based on PI_SM control structure

techniques for the FOC SPIM drive, speed transient time from zero to $1000 \mathrm{rpm}$ at in the case of PI controller is $0.17 \mathrm{~s}$ and $0.1 \mathrm{~s}$, its speed reverse time from $1000 \mathrm{rpm}$ to $-1000 \mathrm{rpm}$ is $1.12 \mathrm{~s}$ in case of PI controller and 1.08s in case of RBF based on PI SM controller. Comparing with the supervisory type-2 fuzzy controller also is made based on recommend [9]. From the survey results show that both controllers have the good speed, rotor flux and torque responses. However, observing the responses of the supervisory type-2 fuzzy controller in [9], we see that the torque and rotor flux responses have higher overshoot and oscillation as shown in [9, Figs. 11-13]. The torque and rotor flux responses oscillate with very large amplitude and only get the stability and convergence with reference value after 5 to 6 cycles. On the contrary, with the RBFPI_SM controller proposed in this paper, the torque and rotor flux are better controlled. The ripple and overshoot of the torque and $i_{\text {sdq }}$ current are very small. The reference value convergence time is very fast, the torque and $i_{\mathrm{sq}}$ current respond almost instantly.

From the simulation results show that the dynamic performance of the vector control for SPIM drive using RBF based on PI _ SM is very good, the real speed follows the reference speed, the speed tracking efficiency is high.

\subsection{The performance of the proposal controllers under change in the speed and the load torque}

In test 2, two cases are surveyed to confirm The performance of the proposal controllers under change in the speed and the load torque.

Case 1 is carried out to verify the robustness of the proposed algorithm when it has to the face of a sudden change of load disturbances based on recommend [8, Figs. 7-10] and [14, Figs. 5 and 7] with the reference speed is kept constant by 1000 $\mathrm{rpm}$ (corresponding to $104.7712 \mathrm{rad} / \mathrm{s}$ ) during the survey process, the rated load torque is applied at $\mathrm{t}=$ $1 \mathrm{~s}$ and rejected at $\mathrm{t}=2.5 \mathrm{~s}$. These proposed controllers give fast and accurate responses but the obtained simulink results of the improved fuzzy controller in [8] and the Fuzzy Sliding mode controller [14] show that the overshoot of the torque, stator current and rotor flux have increased highly (the overshoot of the torque and stator current of the Fuzzy_ Sliding mode proposed controller were $350 \%$, the overshoot of the rotor flux was $150 \%$ recorded in [14], with the improved fuzzy controller in [8], the overshoot of the torque and stator current is lower but higher reference value convergence time). Observing the simulink results in Fig. 6 show that the performance and robustness of the RBFPI_SM controller under the load torque disturbance are better both the controllers proposed in [8] and [14], the RBFPI_SM proposed controller controlled and adapted very well with changing of the load torque. 

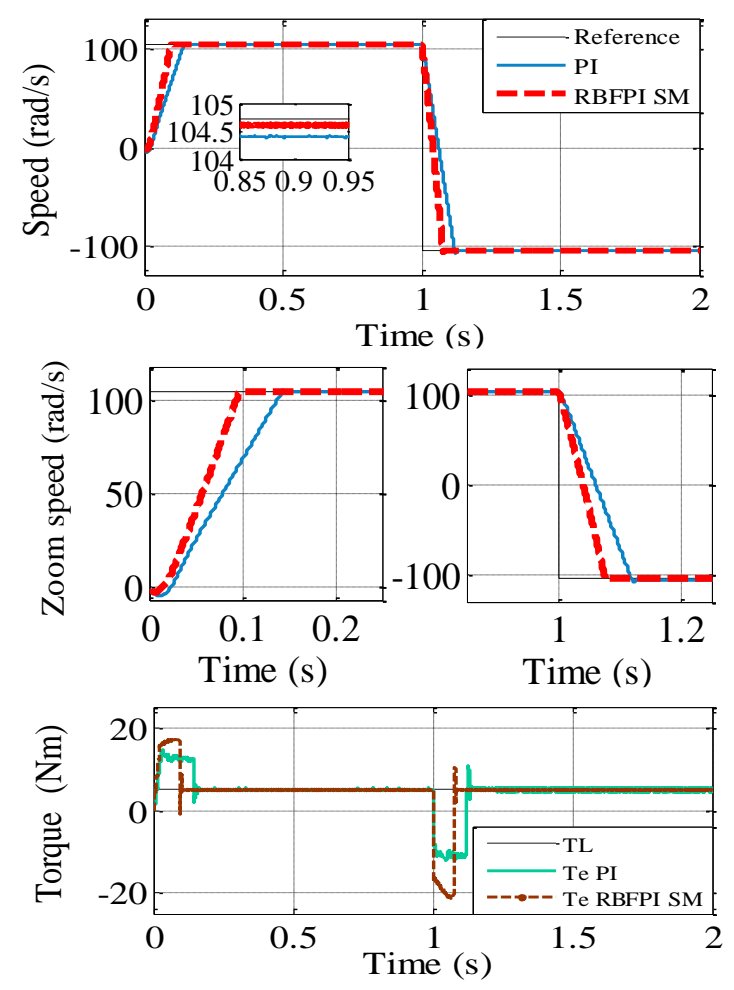

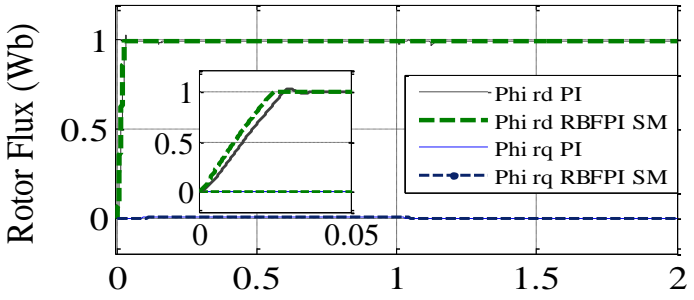

Time (s)

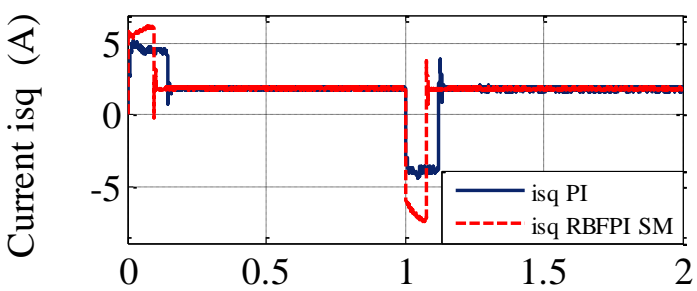

Time (s)

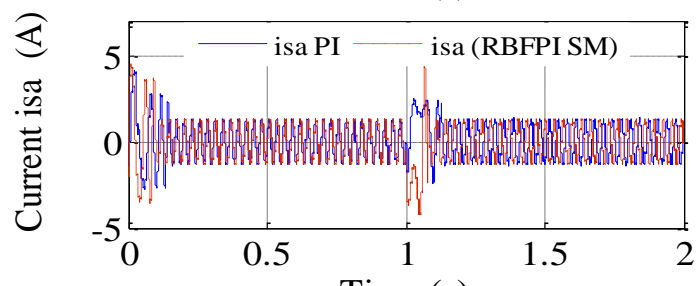

Time (s)

Figure. 5 The dynamic performance of the RBFPI_SM controllers
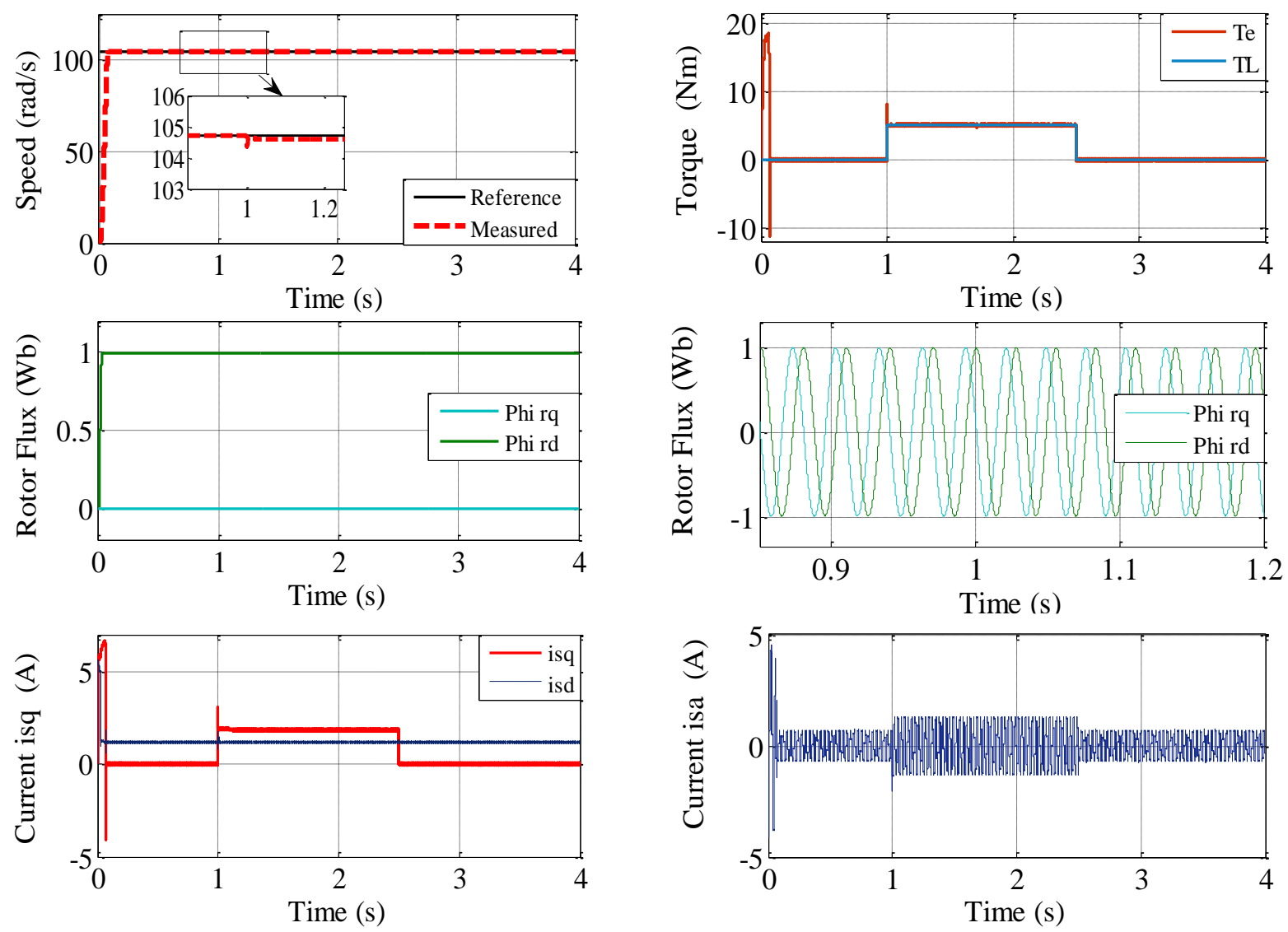

Figure. 6 The performance of the RBFPI_SM controller under the load torque disturbance 

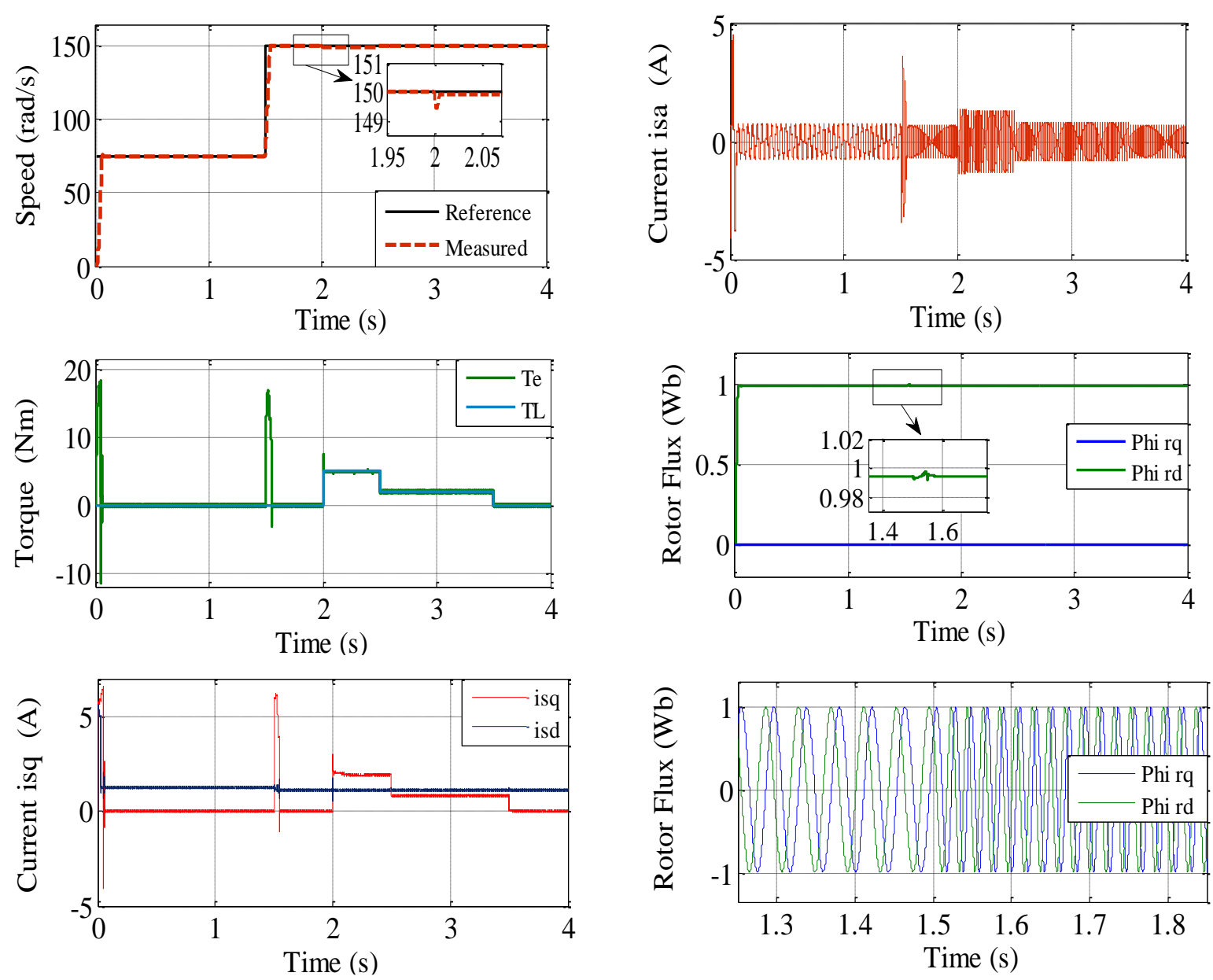

Figure. 7 Simulation results of Indirect Rotor-Flux Oriented Control (IRFOC) of the SPIM in case the torque speed and load variations

Case 2, the reference speed and load torque are changed, the reference speed is changed from 75 $\mathrm{rad} / \mathrm{s}-150 \mathrm{rad} / \mathrm{s}$ at $\mathrm{t}=1.5$, the rated load is applied at $\mathrm{t}=2 \mathrm{~s}$, then being decreased $40 \%$ and rejected at $\mathrm{t}=2,5 \mathrm{~s}, \mathrm{t}=3.5 \mathrm{~s}$, respectively. The results are shown in Fig. 7. Comparing the survey results of the RBFPI_SM controller in Fig. 7 with the survey results of the two controllers proposed in [6, Figs. 3 and 4 ] and [4, Figs. 3 and 4] show that all three proposed controllers have controlled the speed, rotor flux and torque quite well, the speed, isq current, torque and rotor flux responses are fast and follow exactly the reference values. The speed deceleration occurs when applying the load but immediately stabilizing and converging to the reference values. However, when observing the responses of the backstepping controller proposed in [6], we see that the torque, isq current and rotor flux response have higher overshoot and oscillation as shown in [6, Figs. 3 and 4], especially the torque and isq current oscillate with very large amplitude [6, Figs. 3 and 8]. Meanwhile, with the feedback linearizing controller proposed in [4], observing Figs. 3 and 4 in [3] show the high overshoot and torque ripple phenomena, large reference value convergence time, the quality of poor rotor flux controller appear overshoot, oscillation the rotor flux in transient modes (when starting up and changing speed ). With the RBFPI_SM controller proposed in this paper, torque and rotor flux are better controlled, very small torque ripple and overshoot and fast reference convergence time, torque, isq current respond almost instantly. The motor speed follows exactly the reference speed, the speed error in both the transient and steady-state operation are smaller than the two control methods proposed in $[4,6]$.

\subsection{Performance of the proposal controller on step load disturbance}

SPIM is started without load torque until the sudden rated load is supplied at $\mathrm{t}=1 \mathrm{~s}$, rejected at $\mathrm{t}=$ $4 \mathrm{~s}$, the constant reference speed at $125 \mathrm{rad} / \mathrm{s}$. The speed, torque, current, rotor flux responses of SPIM drive are shown in Fig. 8. When applying rated load, undershoot of the PI controller is 1 rad, after $0.038 \mathrm{~s}$ motor speed follows the reference speed. In contrast, 

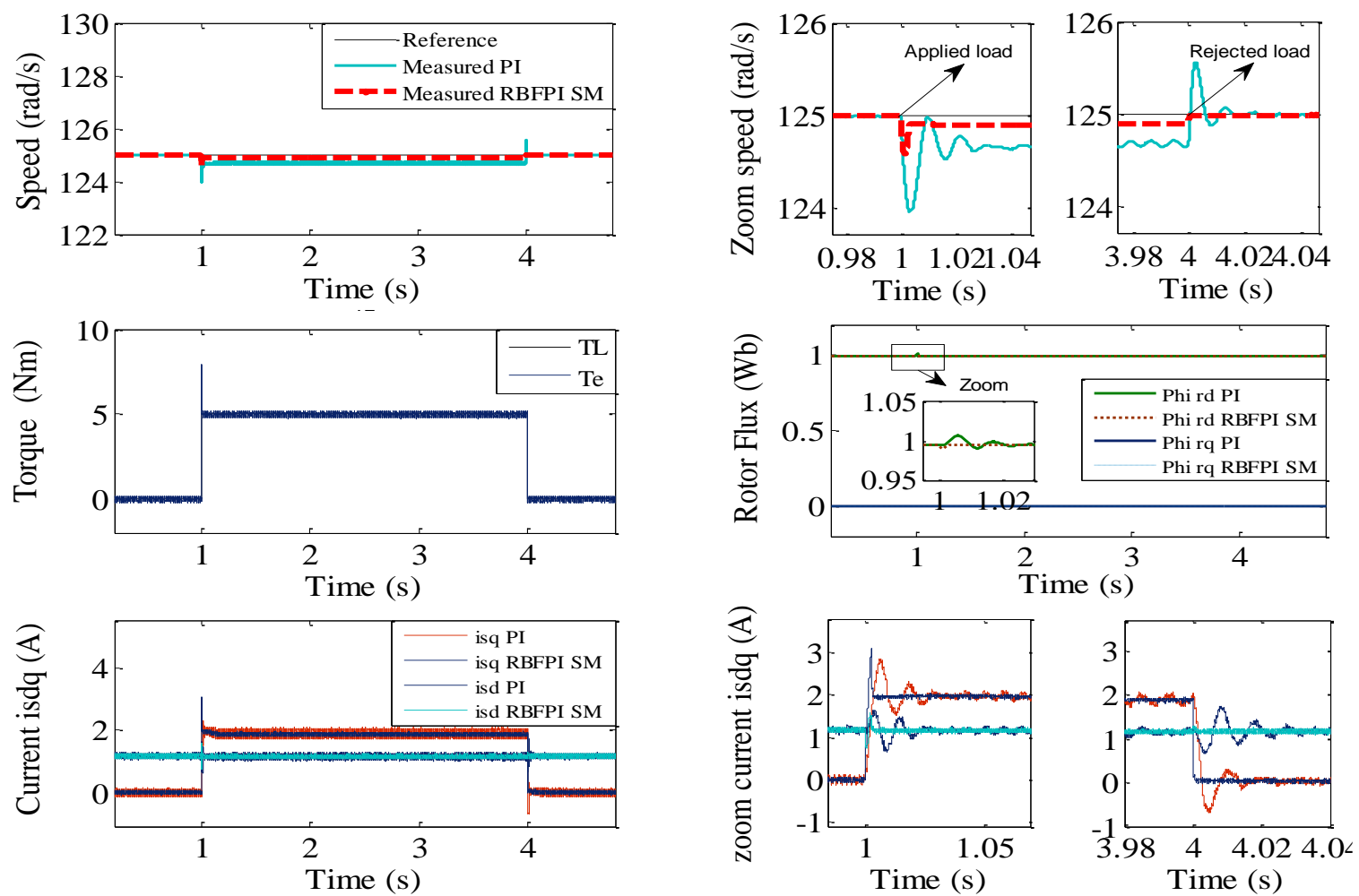

Figure. 8 The performance of the RBFPI_SM and PI controllers under the load torque disturbance
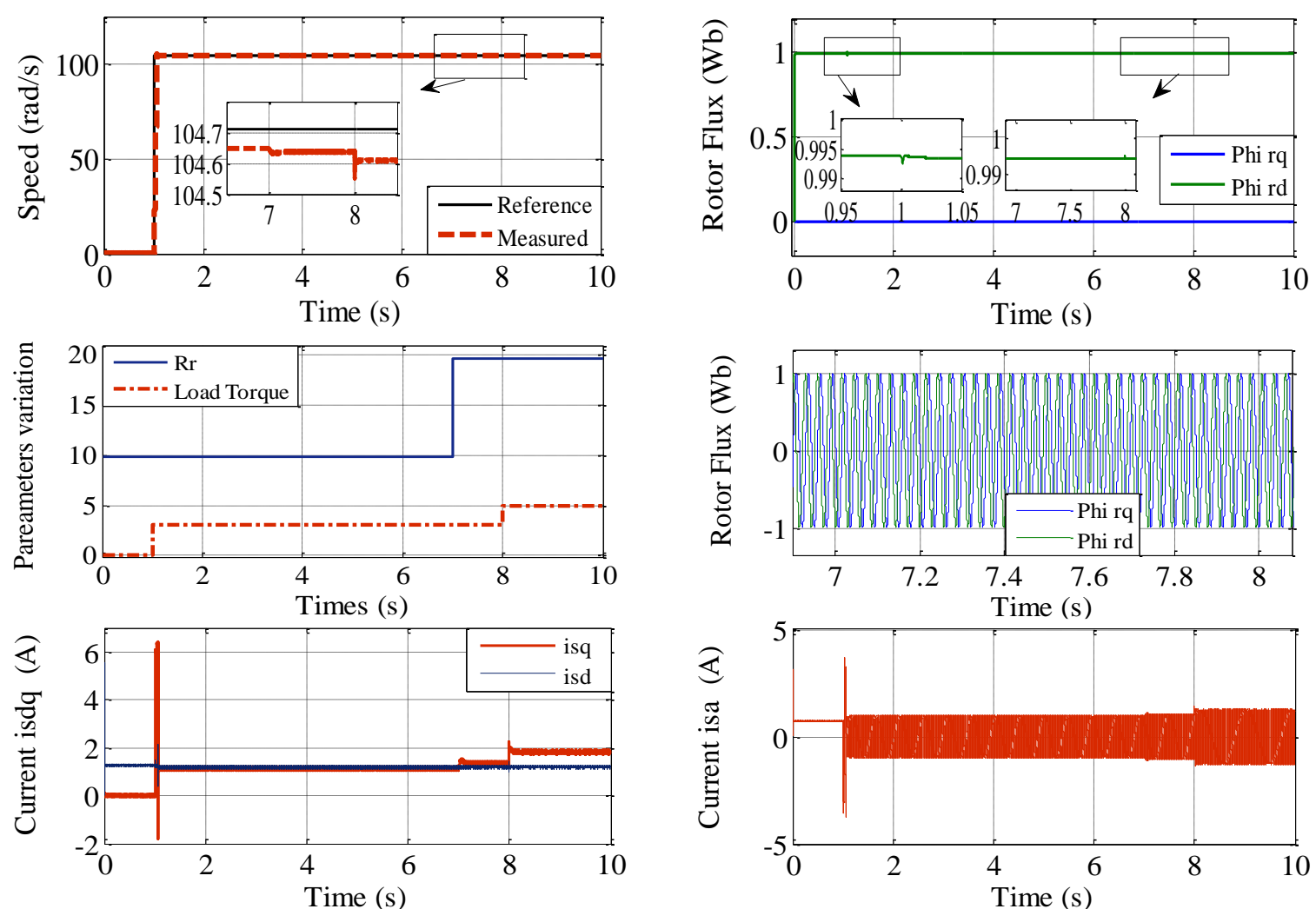

Figure. 9 Simulation results of Indirect Rotor-Flux Oriented Control (IRFOC) of the SPIM in case the load torque and Rs variations

the proposed controller is affected by the load, but almost immediately it follows to the reference speed $(0.0035 \mathrm{~s})$, undershoot of the RBFPI_SM controller is $0.35 \mathrm{rad}$. When rejecting rated load, overshoot of the PI controller is $0.75 \mathrm{rad}$, after $0.04 \mathrm{~s}$ motor speed follows the reference speed, the proposed controller 
adapt very well with the load disturbance, immediately follows to the reference speed, overshoot is zero. Zoom current isq in Fig. 8 shown that tradition PI controller for this current loop has controlled current not good. The rise time, settling time, and recovery time are larger than when using the SM controller for inner current loop, this proposed scheme has controlled isq very well, torque, current response is immediately. Though the simulink results shows in Fig. 8, it is easy to see that the RBF based on PI _ SM controller has a shorter rise time, settling time, and recovery time than PI controller. Also, proposed controller has the faster torque, current response and low torque, current ripple, the characteristic robustness of the RBF based on PI_SM controller to disturbances of the load and the speed variations is better than conventional the PI controller.

To more clarify the effectiveness of the proposed control algorithm, another survey was conducted based on the standard test in [7]. In [7], the FOC vector control uses traditional PI controllers with $\mathrm{Rr}$ updated continue from the $\mathrm{Rr}$ resistor estimators using Mras and Backstepping methods to improve and enhance the SPIM drive's stability when facing with the motor parameter variations and load disturbance. In this test, first, the reference speed and the load torque are kept by 0 , then, at $\mathrm{t}=1 \mathrm{~s}$, the reference speed is and the torque is applied $60 \%$ rated load, the motor works with normal machine parameters. At $\mathrm{t}=7 \mathrm{~s}$, the resistance $\mathrm{Rr}$ is suddenly increased by 2 times its nominal value (Note that, in [7] the survey selected an increase in $\mathrm{Rr}$ by $54 \%$ compared to the initial value of $\mathrm{Rr}$ ). At $\mathrm{t}=8 \mathrm{~s}$, the load torque is increased $40 \%$. From the results obtained in Fig. 9, compared with the survey results in [7, Figs. 5 and 6], it is easy to see that both control strategies have positive results. The system's reliability is greatly improved when facing load disturbances and variable parameters compared to traditional PI controllers. However, in this paper, given the more rigorous survey $(\mathrm{Rr}$ increases $100 \%$ instead of $54 \%$, load increases $40 \%$ instead of $25 \%$ as in [7]), the PI RBF controller is proposed to control the torque, rotor flux and speed better than the strategy proposed in [7]. The proposed controller is almost unaffected by changes in $\mathrm{Rr}$ and load disturbance.

\section{Conclusion}

In this paper, a RBFNN based on PI_SM_novel control structure for FOC vector control of the six phase induction motor drives is presented. The control design is based on combination RBFNN based on PI _SM techniques give more accurate speed control, faster dynamics response, the performance, stability and robustness of SPIM drive system also have been improved significantly. The simulation results and discussion in section 4 confirmed the good dynamics and robustness of the proposed RBFNN based on PI_SM controlled scheme. The comparison data in section 4 also have proven that the RBF_PI_SM controller has controlled the speed better the controlled solutions proposed in [4, 6-9, 14]. However, using RBF neural networks to enhance the performance of the controller will require the computational burden compare to the conventional controllers. In order to improve this problem, the study other NN structures and algorithms, that require less computational effort, will continue to be developed in the future.

\section{Conflicts of Interest}

Declare conflicts of interest or state "The authors declare no conflict of interest."

\section{Author Contributions}

Conceptualization, Ngoc Thuy Pham and Thuan Duc Le; methodology, Ngoc Thuy Pham; software, Ngoc Thuy Pham; validation, Ngoc Thuy Pham and Thuan Duc Le; formal analysis, Ngoc Thuy Pham; investigation, Ngoc Thuy Pham; resources, Ngoc Thuy Pham; data curation, Ngoc Thuy Pham; writing - original draft preparation, Ngoc Thuy Pham; writing - review and editing, Ngoc Thuy Pham; visualization, Ngoc Thuy Pham; supervision, Ngoc Thuy Pham; project administration, Ngoc Thuy Pham.

\section{References}

[1] E. Levi, "Multiphase electric machines for variable-speed applications", IEEE Transactions on Industrial Electronics, Vol. 55, No. 5, pp. 1893-1909, 2008.

[2] J. W. Finch and D. Giaouris, "Controlled AC electrical drives", IEEE Trans. Ind. Electron., Vol. 55, No. 2, pp. 481-491, 2008.

[3] F. Alonge, M. Cirrincione, M. Pucci and A. Sferlazza, "Input-Output Feedback Linearization Control With On-Line MRASBased Inductor Resistance Estimation of Linear Induction Motors Including the Dynamic End Effects", IEEE Transactions on Industry Applications, Vol. 52, No. 1, pp. 254-266, 2016.

[4] A. Sabir and S. Ibrir, "Induction motor speed control using reduced order model", Automatika, Vol. 59, No. 3, pp.275-286, 2018. 
[5] Z. Cheng and L. Jiao, "Hamiltonian Modeling and Passivity-based Control of Permanent Magnet Linear Synchronous Motor", Journal of Computers, Vol. 8, No. 2, 2013.

[6] H. Chaabaneo, K. D. Eddine, and C. Salim, "Sensorless backstepping control using a Luenberger observer for double-star induction motor", Archives of Electrical Engineering, Vol. 69, No.1, pp. 101-116, 2020.

[7] C. B. Regaya, F. Farhani, A. Zaafouri, and A. Chaari, "A novel adaptive control method for induction motor based on Backstepping approach using dSpace DS 1104 control board", Mechanical Systems and Signal Processing, Vol. 100, pp. 466-481, 2018.

[8] A. Saghafinia, H. Wooi Ping, and M. N. Uddin, "Sensored Field Oriented Control of a Robust Induction Motor Drive Using a Novel Boundary Layer Fuzzy Controller", Sensors, Vol. 13, No. 12, pp. 17025-17056, 2013.

[9] H. Rahali1, S. Zeghlache, and L. Benalia, "Adaptive Field-Oriented Control Using Supervisory Type-2 Fuzzy Control for Dual Star Induction Machine", International Journal of Intelligent Engineering and Systems, Vol. 10, No. 4, pp. 28-40, 2017.

[10] X. Fu and S. Li, "A Novel Neural Network Vector Control Technique for Induction Motor Drive", IEEE Transactions on Energy Conversion, Vol. 30, No.4, pp. 1428-1437, 2015.

[11] M. N. Uddin, Z. R. Huang, and A. B. M. Siddique, "Development and Implementation of a Simplified Self-Tuned Neuro-FuzzyBased IM Drive", IEEE Transactions on Industry Applications, Vol. 50, No. 1, pp. 51-59, 2014.

[12] T. Orlowska-Kowalska, M. Dybkowski, and K. Szabat, "Adaptive Sliding-Mode Neuro-Fuzzy Control of the Two-Mass Induction Motor Drive Without Mechanical Sensors", IEEE Transactions on Industrial Electronics, Vol. 57, No. 2, pp. 553-564, 2010

[13] G. Bartolini, E. Punta, and T. Zolezzi, "Approximability Properties for Second-Order Sliding Mode Control Systems", IEEE Transactions on Automatic Control, Vol. 52, No. 10, pp. 1813-1825, 2007.

[14] L. Keltoum, B. Leila, and B. Abderrahmen, "Speed Control of a Doubly-Fed Induction Motor (DFIM) Based on Fuzzy Sliding Mode Controller", International Journal of Intelligent Engineering and Systems, Vol. 10, No. 3, pp. 20-29, 2017.
[15] J. W. Finch and D. Giaouris, "Controlled AC electrical drives", IEEE Trans. Ind. Electron, Vol. 55, No.2, pp. 481-491, 2008.

[16] M. G. Zhang, W. H. Li, and M. Q. Liu, "Adaptive PID strategy based on RBF neural network identification", In: Proc. of International Conference on Neural Networks and Brain, pp. 1854-1857, 2005. 